\title{
DIE RECHTFERTIGUNG DES EHEMALIGEN LANDKOMTURS DER BALLEI ELSASS-BURGUND VON 1443
}

KEYWORDS

Teutonic Order; Ballei Elsass-Burgund; Deutschmeister; Landkomtur; Komtur; Marquard von Königsegg; Ludwig von Landsee; Mainau; St. Georgenschild

国 ie Veröffentlichung des Notariatsinstruments von 1443 lohnt sich, weil es in mehrerlei Hinsicht interessant ist. Zunächst ist die Ortsangabe des Datums bemerkenswert. Das Instrument ist ausgestellt in Stockach in der großen Stube des Hauses Nellenburg am 20. August 1443. Der Ort liegt nicht weit vom Überlinger See, einer Einbuchtung des Bodensees. Die Entfernung beträgt etwa 7 $\mathrm{km}$. Der Ort liegt ferner an der Stockach, die in den Überlinger See fließt. Im Ort Stockach befand sich ein Haus Nellenburg ${ }^{1}$, in dessen heizbarer Stube die Urkunde ausgefertigt worden ist. Dort tagte auch die Gesellschaft vom St. Jörgenschild, an den sich der Komtur der Mainau gewandt hatte. Zumindest vor 1443 zählten die Grafen von Nellenburg zu den Hauptleuten der Gesellschaft. ${ }^{2}$ Ebenfalls waren zahlreiche Angehörige der Familie Königsegg Mitglieder dieser Gesellschaft. ${ }^{3}$ Der Komtur der Mainau, Marquard von Königsegg, trat in die Gesellschaft im Jahr 1442 ein. ${ }^{4}$ nachdem er schon mit der Kommende Altshausen 1434 beigetreten

1 Vgl. H. Seibert, Nellenburg, in: Lexikon des Mittelalters, Bd. 6, München-Zürich 1993, Sp. 1087 f.; Der Landkreis Konstanz, Bd. 1, Konstanz 1968, S. 293 ff.; Bd. 4, Sigmaringen 1984, S. 436 f.; auch Historisch-Biographisches Lexikon der Schweiz, Bd. 5, Neuenburg 1929, S. 241.

2 H. Kruse, W. Paravicini, A. Ranft, Ritterorden und Adelsgesellschaften im spätmittelalterlichen Deutschland (Kieler Werkstücke, Reihe D: Beiträge zur europäischen Geschichte des späten Mittelalters 1), Frankfurt/Main 1991, S. $211 \mathrm{f}$.

3 Vgl. Kruse, Paravicini, Ranft (wie Anm. 2), S. 210-212, 214, 1407-1482, vor allem in den Mitgliederlisten nachweisbar.

4 Kruse, Paravicini, Ranft (wie Anm. 2), S. 212. 
war. ${ }^{5}$ Im übrigen lag die Kommende Mainau nahe an Stockach am Überlinger See. Es wäre verwunderlich, wenn der Mainauer Komtur samt der entsprechenden Kommende dem Bündnis nicht beigetreten wäre. Es ist des weiteren zu erwähnen, dass der Beitritt zu der Gesellschaft dem Mainauer Komtur und der Kommende einen Rückhalt in allen Prozess- und Fehdesachen bot. ${ }^{6}$

Interessant ist auch, dass der Landkomtur Ludwig von Landsee in der sogenannten "mittleren Partei“ in Bad Waldsee geklagt hatte, während sich der Mainauer Komtur Marquard von Königsegg an die „obere Partei“ wandte. Man würde nun voraussetzen, dass es noch eine „untere Partei“ gegeben habe, von der jedoch in der Rechtfertigung Marquards keine Rede war. Immerhin wird man unterstellen müssen, dass die Gesellschaft zum Jörgenschild bereits 1443 in zwei oder gar drei Teile oder „Parteien“ zerfiel. Nach Herbert Obenaus ist schon 1426 eine Dreiteilung der Gesellschaft zu beobachten, nämlich in eine solche des Hegau, der Donau und des Allgäu. ${ }^{7}$ Um welche Teile es sich im vorliegenden Fall gehandelt hat, ist nicht gesagt. Man könnte am ehesten an die Donauregion mit Bad Waldsee und an den Hegau mit Stockach denken. Die „untere Partei“ wäre dann mit dem „Allgäu“ zu identifizieren, ist aber im vorliegenden Dokument, wie schon gesagt, mit keinem Wort erwähnt.

Marquard von Königsegg stammte aus einem schwäbischen Geschlecht mit dem Stammschloss unweit von Ravensburg nördlich des Bodensees. ${ }^{8}$ Er ist 1393 erstmals als einfacher Ritterbruder im Deutschordenshaus Altshausen nachzuweisen. Daraus hat Peter Heim geschlossen, dass er erst kurze Zeit vorher in diese Kommende eingetreten sein könne. Im Jahr 1411 wurde er Landkomtur der Ballei Elsass-Burgund und verwaltete gleichzeitig die Komtursstellen von Altshausen, Mainau und Basel. Er wurde vom Hochmeister mit Verhandlungen unterschied-

5 Kruse, Paravicini, Ranft (wie Anm. 2), S. 212. Die dortige Angabe ist fehlerhaft. Graf Egon von Fürstenberg war kein Landkomtur. Zur angegebenen Zeit war Marquard von Königsegg Landkomtur der Ballei; vgl. Kreuz und Schwert, Mainau 1991, S. 267. Marquard trat mit der Kommende Altshausen in die Gesellschaft ein; K. Militzer, Die Einbindung des Deutschen Ordens in die süddeutsche Adelswelt, in: Ritterorden und Region - politische, soziale und wirtschaftliche Verbindungen im Mittelalter, hrsg. v. Z. H. Nowak (Ordines Militares. Colloquia Torunensia Historica VIII), Toruń 1995, S. 152.

6 H. Obenaus, Recht und Verfassung der Gesellschaften mit St. Jörgenschild in Schwaben (Veröffentlichungen des Max-Planck-Instituts für Geschichte 7), Göttingen 1961, S. 93 ff.; Militzer (wie Anm. 5), S. 152 f.; K. Militzer, Fehde und Deutscher Orden, vornehmlich im Deutschen Reich, Ordines Militares. Colloquia Torunensia Historica. Yearbook for the Study of the Military Orders 18 (2013), S. $184 \mathrm{f}$.

7 Obenaus (wie Anm. 6), S. 197.

8 E. H. Kneschke, Neues allgemeines Deutsches Adels-Lexikon, 8 Bde., Leipzig 1859-1870; hier: Bd. 5, S. 196. 
licher Art, vor allem aber mit solchen während des Basler Konzils betraut. 1434 trat er, wie schon ausgeführt, mit der Kommende Altshausen der Adelsgesellschaft vom St. Jörgenschild bei. Zwei Jahre später, im Jahr 1436, resignierte er krankheitshalber oder altersbedingt das Landkomtursamt und erhielt die Kommende Mainau als Alterssitz. Dort ist er seitdem als Komtur oder als „alter Landkomtur“, also als ehemaliger Landkomtur - bis auf das Jahr 1444 - bezeugt. 1446 ist er gestorben. ${ }^{9}$

An die Stelle Marquards schickte der Hochmeister bereits am 4. Februar 1436 den schon erwähnten Ludwig von Landsee ${ }^{10}$, der ebenfalls einem alemannischen oder schwäbischen Geschlecht mit dem Stammsitz im Thurgau entstammte. ${ }^{11} \mathrm{Er}$ hatte in Preußen mehrere Ämter ausgeübt und dem Orden als Diplomat gedient. Nachdem der Hochmeister am 4. Februar 1436 geschrieben hatte, dass er dem wiederholten Ersuchen des Landkomturs nach einem Rücktritt von dem aufreibenden Amt stattgegeben habe, forderte der Hochmeister am 26. desselben Monats Marquard auf, dem nun ernannten Landkomtur Ludwig von Landsee zu gehorchen. Gleichzeitig beauftragte der Hochmeister den neuen Landkomtur mit der Vertretung der preußischen Interessen auf dem Basler Konzil, insbesondere hinsichtlich der Angriffe des Deutschmeisters gegen die Hochmeister auf Grund der sogenannten „Orselnschen Statuten“. ${ }^{2}$

Ludwig von Landsee war wie Königsegg aus Schwaben und damit aus dem Bereich der Ballei Elsass-Burgund gebürtig. ${ }^{13}$ Zunächst scheinen beide Männer auch gut miteinander ausgekommen zu sein, da sie 1439 noch gemeinsam mit dem Deutschmeister und anderen Gebietigern über Mergentheim nach Frankfurt an der Oder geritten sind. ${ }^{14}$ Jedoch hatte Landsee gegen den zurückgetretenen ehemaligen Landkomtur und nunmehrigen Komtur von der Mainau einen schweren Stand. Denn Marquard von Königsegg beugte sich den Anordnungen Landsees nicht oder nicht immer. Er trat beispielsweise 1442 der Adelsgesellschaft zum Jörgenschild mit der Kommende Mainau gegen den Willen Landsees bei. ${ }^{15}$ Dazu

9 P. Heim, Die Deutschordenskommende Beuggen und die Anfänge der Ballei Elsass-Burgund (Quellen und Studien zur Geschichte des Deutschen Ordens 3), Bonn-Godesberg 1977, S. $151 \mathrm{f}$.

10 K. Neitmann, Ludwig von Landsee. Ein Gebietiger des Deutschen Ordens in Preußen im 15. Jh., Jahrbücher für Geschichte Osteuropas N.F. 36 (1988), S. 182.

11 Kneschke (wie Anm. 8), Bd. 2, S. $491 \mathrm{f}$.

12 Neitmann (wie Anm. 10), S. 182 f. Über Irritationen am hochmeisterlichen Hof über das Auftreten Ludwigs können wir hinweggehen, da sie von Neitmann erschöpfend dargestellt worden sind.

13 E. Weichbrodt, Gebietiger des Deutschen Ordens in Preußen nach ibrer Herkunft (Historischer Atlas des Preußenlandes, Lieferung 1), Wiesbaden 1968; Neitmann (wie Anm. 10), S. 163.

14 Geheimes Staatsarchiv Preußischer Kulturbesitz, Berlin-Dahlem, XX. HA, Schiebl. 99, Nr. 2.

15 Kruse, Paravicini, Ranft (wie Anm. 2), S. 212. 
kamen andere Klagen, die die hochmeisterlichen Visitierer Dietrich von Werdenau, Pfleger von Bütow. ${ }^{16}$ und Konrad, Pfarrer von Rastenburg, dem Komtur Königsegg vorgehalten haben. Zeugen waren fast alle Amtsträger der Ballei ElsassBurgund. ${ }^{17}$

Sowohl Marquard als auch sein Widersacher Ludwig von Landsee reichten den genannten Visitierern schriftliche Klagen über ihren jeweiligen Gegner ein. Die Visitierer erledigten zunächst die Vorwürfe Marquards und gingen dann zu jenen Ludwigs über. Marquard hatte vor allem Geld, und zwar 900 Gulden noch vom Landkomtur zu fordern. Die Visitierer sprachen ihm nur 300 Gulden zu. Ferner hatte Marquard noch 600 Gulden 1 Pfund und 1 Schilling Heller in Sindelfingen vom Grafen von Württemberg zu verlangen. Das Geld solle Marquard zurückgegeben werden, sobald der Graf von Württemberg gezahlt habe. Zahle der Graf zwischen dem 8. April 1442 und dem folgenden 24. Juni nicht, könne Marquard 300 Gulden als Hypothek auf das Haus Mainau aufnehmen. Ein weiterer Punkt war die Stiftung eines Spitals durch Marquard. Darüber entschieden die Visitierer, dass diesen Fall der Hochmeister in Preußen auf dem nächsten Generalkapitel entscheiden solle. Den sechs Punkten, die Marquard vorgebracht hatte, folgten nun insgesamt 23 Klagen des neuen Landkomturs Ludwig. Jenem ging es dabei vor allem um eine Unterordnung Marquards unter dessen neues Amt als Landkomtur. Daher dürfe, wie die Visitierer urteilten, Marquard keine Güter ohne Zustimmung des Landkomturs kaufen, keine Briefe oder Urkunden zurückhalten, nicht den St. Georgenschild gegen den Landkomtur anrufen, müsse dem Landkomtur bei Abrechnungen, bei dem Zuzug zu Kapitelsgesprächen der Ballei, wegen der Reisen außerhalb des Gebiets der Ballei gehorsam sein, solle den Landkomtur ehrbar in der Mainau empfangen, dort keine große Anzahl an Pferden halten und zu den Kosten der Ballei beitragen. Insgesamt haben die Visitierer dem neuen Landkomtur den Rücken gestärkt und dessen Stellung hervorgehoben, wie es die Statuten des Ordens vorschrieben. Allerdings durfte Marquard seinen Alterssitz in der Mainau behalten, musste aber auf seine Stellung als ,alter Landkomtur" zugunsten Ludwigs von Landsee verzichten.

16 B. Jähnig, Wykaz urzędów, in: Państwo zakonu krzyzackiego w Prusach, hrsg. v. Z. H. Nowak, Toruń 2000, S. 118, hat Dietrich von Werdenau nur zum Jahr 1441 nachgewiesen. Er muss aber wenigstens bis 1442 Pfleger von Bütow gewesen sein; vgl. Regesta historico-diplomatica Ordinis S. Mariae Theutonicorum 1198-1525, bearb. v. E. Joachim, hrsg. v. W. Hubatsch, Pars I: Regesten zum Ordensbriefarchiv, Vol. 1-3; Göttingen 1948-1973; hier: Vol. 1, Nr. 2548, 2555.

17 Visitationen im Deutschen Orden, Teil I: 1236-1449, hrsg. v. M. Biskup, I. Janosz-Biskupowa (Quellen und Studien zur Geschichte des Deutschen Ordens 50, Veröffentlichungen der Internationalen Historischen Kommission zur Erforschung des Deutschen Ordens 10), Marburg 2002, Nr. 105; Regesta (wie Anm. 16), Pars. 2: Regesten der Pergament-Urkunden aus der Zeit des Deutschen Ordens, Göttingen 1948, Nr. 2548; Heim (wie Anm. 9), S. 152. 
Offenbar hat der Hochmeister die Vorgaben der Visitierer genehmigt. Jedenfalls hat er sich noch 1442 bei dem neuen Landkomtur der Ballei ElsassBurgund für die erfolgreiche Visitation bedankt. Auf der Rückseite des Konzeptes ist vermerkt, dass der Hochmeister die Auseinandersetzungen durch den Spruch der Visitierer für beendet hielt. ${ }^{18}$

Der im obigen Notariatsinstrument geschilderte Streit zwischen Ludwig von Landsee und Marquard von Königsegg soll sich vor allem wegen einer vom Hochmeister geplanten Einlösung der Ballei Elsass-Burgund durch den Deutschmeister erhoben haben. Letzterer hatte die Ordensprovinz im Jahr 1386 zu Pfand gesetzt und zwei Jahre später nicht wieder eingelöst. Seitdem blieb die Ordensprovinz eine der Kammerballeien des Hochmeisters. Obwohl nun der Deutschmeister Eberhard von Seinsheim dem Orden in Preußen und dem Hochmeister erhebliche Summen hatte zukommen lassen, blieb die Ballei der hochmeisterlichen Kammer zugeordnet und nicht zuletzt deshalb, weil die in den Kommenden der Provinz lebenden Ordensbrüder es so wünschten. Denn ihr Sonderstatus erlaube es ihnen, Brüder auch aus anderen Gebieten anzuwerben und damit weitere Mittel zu bekommen, als wenn sie wie die übrigen benachbarten Balleien dem Deutschmeister und nicht mehr direkt dem Hochmeister unterstünden. ${ }^{19}$ Dazu traten außenpolitische Probleme, die teilweise wohl vom Deutschmeister hochgespielt worden sind. Es ging um den Frieden von Brest vom 31. Dezember 1435, an dem Ludwig von Landsee beteiligt gewesen ist, auch wenn er angeblich nach Aussage des Deutschmeisters eine Teilnahme oder Zustimmung zu dem Frieden nicht gegeben bzw. später eine solche geleugnet habe. ${ }^{20}$ Dagegen spielten die sogenannten „Orselnschen Statuten“, die der Deutschmeister Eberhard von Seinsheim gegen den Hochmeister Paul von Rusdorf vorgebracht hatte, keine Rolle mehr. ${ }^{21}$ Wie wir noch sehen werden, kamen ordensinterne Probleme hinzu, die mit dem Rücktritt Königseggs vom Landkomtursamt zusammenhingen.

Über die von den Visitierern festgehaltenen Verfehlungen sprach Marquard von Königsegg laut dem Notariatsinstrument von 1443 nicht. Es ging ihm in dieser Hinsicht vielmehr um zwei Dinge. Erstens meinte er, dass Ludwig von Landsee

18 Visitationen, Tl. 1 (wie Anm. 17), Nr. 112.

19 K. Militzer, Die Entstehung der Deutschordensballeien im Deutschen Reich (Quellen und Studien zur Geschichte des Deutschen Ordens 16), Marburg ${ }^{2}$ 1981, S. 158 f.; vgl. auch B. Demel, Der Deutsche Orden und seine Besitzungen im südwestdeutschen Sprachraum vom 13. bis 19. Jahrhundert, Zeitschrift für württembergische Landesgeschichte 31 (1972), S. 32 ff., 52 ff.

20 Vgl. Neitmann (wie Anm. 10), S. 161-190.

21 D. J. Weiss, Die Geschichte der Deutschordens-Ballei Franken im Mittelalter (Veröffentlichungen der Gesellschaft für fränkische Geschichte, Reihe IX: Darstellungen aus der fränkischen Geschichte 39), Neustadt/Aisch 1991, S. 292 ff. 
vor ihm weltliche Institutionen angerufen ${ }^{22}$ und er selbst nur in zweiter Linie dasselbe getan habe, wie ihm sein Beistand Simon von Leonrod, Komtur der Kapfenburg, geraten habe. Im übrigen habe er den Vorwürfen der Visitierer in einem Brief an den Hochmeister entgegnet, wie es auch der Marschall von Pappenheim getan habe. ${ }^{23}$ Zweitens wehrte er sich dagegen, dass er dem Deutschmeister die Einlösung der Ballei empfohlen und sogar Geld dafür gereicht oder zumindest in Aussicht gestellt habe. Letzteres habe er jedenfalls nicht getan, wie jeder, der ihn kenne, wisse.

Nach dem Generalkapitel in Preußen wurde dem Deutschmeister offenbar angedeutet, dass die Ballei Elsass-Burgund eingelöst werden könne. Der Komtur von Sumiswald, Rudolf von Rechberg ${ }^{24}$, ritt vom Deutschmeister zu den Gebietigern der Ballei, die sich aber nach der Aussage Marquards an ihn selbst wandten, da er die meisten Erfahrungen habe. Marquard riet, dass die Gebietiger ihre Häuser und Schlösser besetzten und wohl bewahrten, indem sie keinen hereinließen.

Anschließend muss Marquard 1442 nach Beuggen zu einem Balleikapitel oder einem anderen "Gespräch“ geritten sein, wie der sogenannte „Doktor in Preussen " ihm ${ }^{25}$ und anderen geschrieben habe. ${ }^{26}$ Der in der obigen Urkunde genannte Doktor von Preußen, der ihm befohlen habe, nach Beuggen zu reiten, mag mit dem Visitierer Konrad, dem Pfarrer von Rastenburg, identisch sein. Damals ist jedenfalls Marquard nach Beuggen gezogen und hat sich dort vor den Visitierern gerechtfertigt. ${ }^{27}$ Ferner habe jener „Doktor“ dem jungen Türing von Hallwil, aus der heutigen Schweiz aufgetragen, die Mainau zu besetzen und niemanden hereinzulassen. Dass jener Türing ein Bruder des Deutschen Ordens gewesen sei, ist unwahrscheinlich. ${ }^{28}$ Jedenfalls ist 1444 ein Türing von Hallwil, der Junge, als

22 Landsee habe sich vor ihm in Bad Waldsee, offenbar an den St. Jörgenschild, gewandt. Dabei kann es sich wohl nur um den Teil der Donauregion gehandelt haben.

23 Die Briefe sind anscheinend nicht erhalten.

$24 \mathrm{Zu}$ der Familie mit dem Stammsitz etwa $8 \mathrm{~km}$ südlich von Schwäbisch-Gemünd entfernt vgl. Historisch-Biographisches Lexikon der Schweiz (wie Anm. 1), S. 549; Kneschke (wie Anm. 8), Bd. 7, S. 374 ff.; Europäische Stammtafeln, N.F., hrsg. v. D. Schwennicke, Bd. 5, Marburg 1988, Tafel 89. Zu Sumiswald zuletzt: B. Häfliger, Der Deutsche Orden in der Schweiz, in: Der Deutsche Orden und die Ballei Elsaß-Burgund, hrsg. v. H. Brommer, Bühl 1996, S. 281; auch Historisch-Biographisches Lexikon der Schweiz (wie Anm. 1), Bd. 6, S. 607.

25 Mit dem „Doktor“ könnte einer der Visitierer, also wohl der Pfarrer von Rastenburg gemeint gewesen sein, aber das bleibt ungewiss.

26 Wer damit gemeint sein könnte, ist nicht ganz klar. Es dürfte sich um Heinrich Marschall von Pappenheim gehandelt haben: Europäische Stammtafeln, N.F., hrsg. v. D. Schwennicke, Bd. 4, Marburg 1991, Tafel 55.

27 Visitationen, Tl. I: (wie Anm. 17), Nr. 105.

28 Nach Historisch-Biographisches Lexikon der Schweiz (wie Anm. 1), Bd. 4, S. 63, war ein Türing von Hallwil, der Jüngere, der Sohn Türings von Hallwil, des Älteren, 1444 Ritter. Türing der 
Mitglied der Gesellschaft des St. Jörgenschildes verzeichnet. ${ }^{29}$ Immerhin scheint die Angelegenheit zugunsten Marquards erledigt worden zu sein. Denn er kam wieder in den Genuss der Komturswürde. Allerdings ist ein Beringer von Weiler, der 1442 noch Komtur von Freiburg war, 1444 als Komtur der Mainau geführt worden. ${ }^{30}$ Ludwig von Landsee scheint, dagegen schon am Ende des Jahres 1442 die Würde eines Landkomturs von Elsass-Burgund gegen die der Kammerballei Bozen eingetauscht zu haben. ${ }^{31}$ Wahrscheinlich ist er vom Hochmeister zu dem Wechsel gedrängt worden. 1450 ist er dann als Landkomtur gestorben. ${ }^{32}$

Der neue elsässische Landkomtur Burchard von Schellenberg, der am 23. November 1443 vom Hochmeister bestätigt wurde ${ }^{33}$, ließ Marquard von Königsegg anscheinend weitgehend in Ruhe, nachdem am Anfang des Jahres 1444 noch einmal ein Komtur der Mainau ernannt worden ist, eben jener Beringer von Weiler. Bis zum Tod Königseggs sind daher keine weiteren Komture der Mainau nachzuweisen. Es hat sie auch wohl nicht gegeben. Königsegg hat das Ordenshaus vielmehr weitgehend ungestört als seinen Alterssitz verwaltet.

Marquard von Königsegg hat seine Würde trotz seines Rückzugs nie ganz aufgegeben. Er war im übrigen in der Ballei und mit den führenden Kräften der Region gut vernetzt, ein Vertreter der auf ihrem Ansehen oder ihrer „Ehre“, wie sie es nennen mochten, achtenden Adligen, gleichgültig ob sie nun in einen Orden eingetreten waren oder weltlich geblieben sind. Ludwig von Landsee dagegen hatte trotz aller gegenteiligen Erscheinungen im Preußenland den Gehorsam ge-

Jüngere ist 1444 auch in den Listen des St. Jürgenschildes erwähnt; Kruse, Paravicini, Ranft (wie Anm. 2), S. 213. Jener Türing, der Jüngere, käme also in Frage.

29 Kruse, Paravicini, Ranft (wie Anm. 2), S. 213.

30 Kreuz und Schwert (wie Anm. 5), S. 268; vgl. Heim (wie Anm. 9), S. 183 f., der die Nachweise über Beringer gesammelt hat. Danach war er nur am 7. Februar 1444 Komtur der Mainau. Nach Heim entstammte er einem ehemaligen sanktgallischen Dienstmannengeschlecht bei Lindau am Bodensee.

31 Neitmann (wie Anm. 10), S. 185; J. Ladurner, Urkundliche Beiträge zur Geschichte des deutschen Ordens in Tirol, Innsbruck 1861, S. 100; U. Arnold, Mittelalter, in: Der deutsche Orden in Tirol, hrsg. v. H. Noflatscher (Quellen und Studien zur Geschichte des Deutschen Ordens 43), Bozen-Marburg 1991, S. 155, 522

32 Arnold (wie Anm. 31), S. 155, 522. Bereits am 9. Mai 1451 schrieb der Hochmeister der Ballei Bozen wegen der Neubesetzung des Landkomtursamts der Ballei Bozen. Daraus schließt Neitmann, dass Landsee erst 1451 in seinem Amt gestorben sei; Neitmann (wie Anm. 10), S. 185, Anm. 150. Angesichts der langen Laufzeit für Briefe an den Hochmeister und von ihm und der Zeit der Beratungen, wird man wohl das Ende 1450 als Todesdatum annehmen müssen.

33 Burchard von Schellenberg aus Bayern oder dem heutigen Liechtenstein war zunächst seit 1428 Komtur von Beuggen gewesen, ehe er 1443 zum Landkomtur der Ballei Elsass-Burgund aufstieg; Heim (wie Anm. 9), S. 180 ff. Ob er 1453 für wenige Monate das Amt eines Deutschmeisters bekleidet hat, wie Peter Heim meint, mag dahin gestellt sein. 
genüber den Vorgesetzten oder „Oberen“, wie sie es nannten, erlebt und wollte dergleichen auch in der Kammerballei Elsass-Burgund durchsetzen. Letztendlich ist ihm das nicht gelungen. Ludwig wurde abgelöst und mit der Ballei Bozen abgefunden, während der neue Landkomtur der Ballei Elsass-Burgund aus der Region selbst kam und darin groß geworden ist. Jener ließ Marquard von Königsegg, dem „alten Landkomtur" in dessen Alterssitz, der Kommende Mainau, weitgehend freie Hand.

\section{Quellenanhang}

Stockach im Bistum Konstanz,

Neues Haus, genannt Nellenburg, oben in der großen Stube, 20. August 1443

DOZA Wien, Urkunden - Notariatsinstrument - Ausf. Perg. Dt. - 55,1-57,3x 39,2-42,4cm. - Notariatssignet - Unbesiegelt und obne Rückaufschrift.

Regest: Die Urkunden des Deutsch-Ordens-Centralarchives zu Wien, hrsg. von Ed. Gaston Grafen von Pettenegg, Prag-Leipzig 1887, Nr. 1956; Die Urkunden des Deutschordens-Zentralarchivs in Wien. Regesten, Bd. 3, hrsg. v. Udo Arnold (Quellen und Studien zur Geschichte des Deutschen Ordens 60/III, Veröffentlichungen der Internationalen Historischen Kommission zur Erforschung des Deutschen Ordens 11/III), Marburg 2007, Nr. 3491.

In Gottes namen amen.

Nach gepůrt Cristi, unsers herrn, vyerczehen hundert und jm drü und vyerczigesten jär, des sechsten järs der römerczal, do das hailig concily zu Basel dennocht weret, an dem czwainczigosten tag des manocz genannt Augustus umb mittentag des selben tages oder ain wenig darnach zu Stockach jn der statt, Costencz bystům uff dem nüwen huse genannt der von Nellenburg huse oben jn der grössen stuben jn gegenwürtikayt vil löblicher gaistlicher und weltlicher herren und personen der gesellschafft und ritterschafft der obern parthye in Swaben rett herr Marquart von Kungsegg, commentür jn der Mayenöw, Costenzer bystům, jn gegevürtikait mins hier under geschriben offenn schribers und gezügen, wye man wol vernommen hett sollich zwayunge, so zwüschen jm und dem von Lönse, der sich nampt lanndcomentür jn Elsäß, des er doch nitt wär, ufferstannden waren. Das wär jm nu layd von des ordens wegen, wann er hette sust ungern mit yeman zwayung und hett die noch ungerner mit den, so jn seinem orden wären. Nu wär aber er des nit ain an- 
heber gewesen söllich sach für weltlich ze tragen, sonnder der von Lonse hett des für ain gesellschaft der mitteln parthy zu Walse fürgetragen. Das wöllt er davor der gesellschafft und ouch andern und, so ye mer darby wären, so jm ye lieber wär, ye verantworten. Darby man wol verstünde, welher tayl an dem andern ungelimpfet hette, und rett füro mit sinem redman, den commentur zu Kapffemburg, wye das ain manung gewesen wär zu Walse. Da hetten herr Ludwig von Lönse, der ainest lanndcommentur jn Elsaß gewesen und yetz nymmer wär, und ettlich gepietiger mit jm da fürgetragen und lesen lässen ainen richtungbrieff, so dann die visityerer und gepietiger gemachet hetten und darzu abgeschrifften söllich brieff. So dann der commentur von Mayenöw und ouch ettlich sin fründ ainem hochmaister gen Prüssen geschribenn hetten und dar jn nu der Lonse gerett hett synttemal und der brieff datum gelich seyt. Dadurch man wol vergrän möchte, das sollich brieff etwas gevarlich zugegangen waren. Da wär geschriben worden von sinen fründen und hett da ainer versigelt für die andern und wye das zugegangen wär, wer das wissen wöllt, der mocht söllichs erfarn an den, die söllich brieff versegelt hetten. Die wißten das wol zu verantlorten [!], und ueff das batt er, zwen brieff verhören und daruff sine wort, und was ain brieff, den er dem hochmaister gen Prüssen geschriben hett zu dem grössen capitel, der ander, so der marschalk zu Bappenhain ouch hin jn geschriben hetten von sinen wegen, und rett daruff, wye man wol verstünd an den geschrifften und brieffen, wie er der sach uff sine obern zekommen begert hette. Uber das hette der von Lönse söllichs des ersten für weltlich bracht und er nitt.

Item als der maister von Tütschen lannden by dem grössen cappitel gewesen sye und die gebyet und baly zu Elsäß ervordert habe ze lösen von dem hochmaister etc. durch ain vidimus als die visitatores höptbrieff und gelöblich vidimus mit zwayer byschoff jnsigeln besigelt genainander verlesen und das vidimus dem hochmaister zu Prüssen etc. fürbracht haben. Nu gäbe der von Lonse uß, wye der commentur jn der Mayenöw gelt geben hett ainem mayster Tütsches lanndes, das die selb lösung beschäch etc. Das hett er nit getön, wann er wär also herkommen, das man jn darfür hett, das er sins geltz so milt nit wär ußzegeben, er wißte dann, das es sin nucz wär. Nu hette er doch gepetten, jn des lanndcommentur amptz ze erlässen krankhayt halb sins libs, und hett darvon gestellt, dardurch man wol verstän möcht, das er söllicher hilff der lösung unschuldig wär. Also hett nu der hochmaister etc. ainen doctor heruß gesennt umb söllichen höptbrieff ze ervordern und den dem von Lanse und gepietiger ze zögen, der aber der mayster von Tütschen lannden nit tůn wöllt. Dann man wol verstän möcht, das jm söllich nitt ze tünd wär, nach dem und er söllich gelt geben und lösung geton hette und jm söllich gepyet noch nitt jn geantwurt wär. Doch hatt sich der mayster von Tüschen lannden sälig erbotten, den selbenn höptbrieff ze sennden gen Ulm und ander ge- 
legen ende, da er des sicher maynt ze sin, und den da selbs ze hören und ze lesen ze lässen.

Item söllich lösung der baly zu Elsäß her Ludwig von Lönse verwilget hette vor dem hochmaister und andern, daruff er ouch des lanndcomentür ampt zu Elsäß entsetzt und jm das lanndcomentür ampt ze Bötzen an der Etsch enpholhen wäre, und jm der hochmaister schrib lanndcomentur zu Bötzen und nit ze Elsäß. Uff söllichs er billich dasselb lanndcomentür ampt zu Botzen jngenommen und das lanndcomentür ampte zu Elsäß unbekumert gelässen hette. Aber wie dem allen, so habe der hochmaister etc. jn bayden tag für sin gnäd gesetzt, herumb so gebůr jm nit, zu der sach vil ze reden. Hette es aber ain ander gestalt, so hette er vil mer und tyeffer zu den sachen zu reden, das er aber yecz zemal nitt tün welle, dann er hett willen für den hochmaister etc., als jm geschriben wär und die sachen daselbs ußzetragen.

Item als das größ cappitel zu Brüssen ain ende hette, also ritte der von Rechberg, comentür zu Sumißwald vor dem maister Tütsches lanndes heruß zu den gepietigern der baly zu Elsäß und leyte jn söllich lösung für. Uff das kamen ettlich gepietiger hin jn gen Mayenöw zu dem commmentür daselbs und leyten jm söllichs ouch für und hetten sinen raitt dar jnn, wann er doch lang zit by dem orden und jn der gepyet gewesen wär und jm mer umb söllichs ze wissent wär dann jn. Also rett er mit jn und wär sin raute und maynung, das man die hüser bewaret und bemannet, besunder die schloß, als sy ouch ettliche bewarten, des gelichen wölt er ouch tün.

Da gepurt jm nu zü ainem tag ze riten gen Büky, als der doctor von Prüssen jm und andern geschriben hette, und befülhe dem jungen Türing von Halwig das huß Mayenöw innzehaben und nyeman jn zelässen und ob er sturb oder wye es umb jn ergieng, so söllte er das huse jnnehaben und bewaren ze ains hochmaisters etc. handen. Also wären komen ettlich gepietiger für die Mayenow, da wöllt der von Halwig ain söllichs enphelhencz wegen nyeman hin jn lässen.

Item der von Lonse hett ouch nach des maisters Tütsches lannds töd vil uff den selben mayster geschriben, als er vernam. Bedüchte jn, er hette das billicher getan by des maisters säligen leben dann nach sinem töd, wann doch der von Lonse vor des maisters töd etwe vil zits sol gewisset hett, was der maister des Tüschen lannds sälig von jm geschriben hette. Beduchte jn, er hette es billicher verantwort by sinem leben. Ouch saczt er zu letzt durch den jungen Turing von Halwig, was er da für getragen hett laussen, das hett er nit getan jn klag wise und wöllt ouch nit klagen. Dann wär es also gestalt, das er klagen söllt, so wöllt er vil tyeffer und mer laussen reden. Das wöllt er aber sparen uncz an die ende. Dahin er gerüfft wär, da wöllt er ouch hinkommen.

Daruff rett her Marquart obgn[ant] mit mir, dem notary hier under geschriben, was ich dar wyder reden wöllt. Antwurt ich, mir wär nit mer enphalhen von 
mins herrn des lanndcomentürs gnäden, dann zu zehören, und hett des gemerkt, als vil ich dann hett mögen begriffen, wann der wären sachen erzelt, die mir vor unkund wären und nye mer gehört hette. Rette er, ob ich dem von Lonse darumb jnstrument machen wöllt. Antwurt ich, ob sin gnäd des begert, so wöllt ich jm darumb jnstrument machen, als vil jch dann behept hett, und wöllt darjnn minen beßten fliß tün. Da begert er ouch ains jnstruments von mir, da jnn die sachen aigenlich begriffen wären, wye dann das fürgetragen wär worden. Antwurt ich, das er zů mir saczt sinen redman, der mir die sach angäb, als er die gerett hett, so wöllt ich das seczen vor erbern gezügen, das ich hoffte, das ich recht tät. Antwort er, das er das nit tůn wöllt. Ich söllte es selber begriffen und jm das ain jnstrument geben. Antwurt ich jm als vor.

Darnach kam er wyder für die geselschafft und batt, jm söllichs fürtragens urkund zu geben. Dagelich begert ouch ich von mins herrn des lanndcomentür jn Elsaß jm ouch urkünd zu geben. Begert der ritterschaft schreiber jm die sach ouch jn geschrifft ze geben. So wöllt er daruff urkund machen, und wann minem herrn herrn Ludwigen von Lönse lanndcomentur jn Elsaß etc. umb söllich fürgetragen sachen von der obgn[anten] loblichen herrschafft der obern parthy obgn[ante] urkunde noch nit worden ist, so hät mich sin gnad ervordert mit flissigem ernst und ermant mines amptz, als hoch er kund, jm der sachen, als vil ich der wisse und behept habe, offenn urkunnd und ains oder mer tütsches jnstrument ze geben. Und wann man der warhait billich züknüß geben sol und mir das gepürt von ampcz wegen, so hän ich diß jnstrument darvon gemachet und mit flissigem ernst darjn geschrieben, wye und als vil jch der fürtragung behept, gemerkt und verstanden hän. Und ist das geschehen des järs, der römerczal, werunge des concily, des manotz, des tags, der stund und an den stetten und enden, als obgeschriben ist. Darby und mit synd gewesen die erbern Hainricus, schriber, des alten stattschribers in Ravenspurg säligen sun, Thoman, des commentürs von Bern, und Hanns von Tengen, des commentürs von Büken diener, die zugezügen der vorschr[iben] sach ervordert und gebetten synd worden.

Und ich, Petrus Kungschlacher von Ingelstat, Aistetter bystum, zu den ziten schůlmaister zů Sulgen, Costenzer bystum, wann ich by der obgeschriben fürtragung und rede mit sampt den vorgeschriben zügen personlich gegenwürtig gewesen bin, gesehen und gehört hän, herumb so hän ich diß jnstrument darvon gemachet und mit miner aigen hand geschriben und mit minen gewohnlichen namen und zaichen bezaichnet, wann ich, als vorstät, zu gezüknuß aller ${ }^{34}$ und yeglicher vorgeschr[iben] sachen gerüfft und gebetten bin worden.

\footnotetext{
${ }^{34}$ Verbessert aus: alber.
} 


\section{Summary \\ The justification of the former Landkomtur of the bailiwick of Alsace-Burgundy from 1443}

When Maquard von Königsegg resigned his Landkomtur's office and received Mainau as a provision for his old age, he got in conflict with the new Landkomtur Ludwig von Landsee sent by the Grand Master of the Order. The conflict concerned the obedience of the old and new Landkomturs according to the Order's rule, as well as the opening of a hospital in Mainau, the joining of St. Georgenschild and other matters of lower importance. Although visitors sent by the Grand Master mostly confirmed the opinion of Ludwig von Landsee, he seems to have been relieved from his post and sent to the bailiwick of Bozen. Nevertheless Marquard von Königsegg was never appointed Landkomtur again even though he justified his position in a letter addressed to a range of officials including the Grand Master in Prussia. 